\title{
Wer Optimismus trainiert, erträgt Schmerzen besser
}

\section{Eine internetbasierte Intervention aus dem Bereich der so ge- nannten Positiven Psychologie soll Patienten mit chronischen Schmerzen zu einem erträglicheren Leben verhelfen.}

\) Die Positive Psychologie will sich von der angeblich defizitorientierten traditionellen Psychologie abgrenzen und sich stattdessen jenen Aspekten des Lebens zuwenden, die den Menschen stärken, das Befinden heben und allgemein die Lebensqualität steigern. Niederländische Psychologen um Madelon Peters, Universität Maastricht, haben untersucht, ob sich auf dieser Grundlage auch das Leben von Patienten mit chronischen Schmerzen lebenswerter gestalten lässt. Dabei verwendeten sie ein internetbasiertes Interventionsprogramm mit dem Titel „Happy Despite Pain“ (HDP).

Teilnehmer der Studie waren 276 Patienten mit chronischen, im Mittel seit knapp 13 Jahren andauernden, beeinträchtigenden muskuloskeletalen Schmerzen. Sie wurden randomisiert entweder dem HDP-Programm oder einer ebenfalls internetbasierten Intervention mit kognitiver, auf verbesserte Schmerzbewältigung gerichteter Verhaltenstherapie zugeordnet. Die Kontrollgruppe bildeten Patienten, die einstweilen auf eine Warteliste für eine der beiden frei zu wählenden Behandlungsformen gesetzt worden waren.
Die HDP sollte die Einstellung sich selbst gegenüber verbessern und positive Gefühle sowie Optimismus stärken. Das Training dafür ging über vier Stufen.

\section{Mitgefühl mit sich selbst üben}

Zunächst wurde zwei Wochen lang das Mitgefühl für die eigene Person geübt, um mehr Selbständigkeit im Umgang mit den emotionalen Folgen der chronischen Schmerzen zu erlangen. Dabei helfen sollten etwa ein Mantra sowie ein mitfühlender Brief an die eigene Adresse. In der Woche darauf folgte die Übung „Drei gute Dinge“. Um statt einer negativen eine positive Orientierung zu erlangen, war täglich Buch zu führen über drei Dinge, die gut gelaufen waren, und über die Gründe hierfür. Hierauf galt es, zwei Wochen lang Genusstechniken einzuüben, die auf kleine Annehmlichkeiten des Alltags gerichtet waren. Woche 6 und 7 waren dem „Bestmöglichen Selbst“ gewidmet. Die Patienten sollten sich ein gutes Leben trotz der Schmerzen vorstellen und Ziele sowie Ideale für verschiedene Lebensaspekte formulieren.
Im Vergleich zu den Patienten auf der Warteliste war die HDP durchaus erfolgreich. Was Depressionen und Glücksempfinden anging, gab es statistisch betrachtet starke Vorteile für die Interventionsgruppe. Keine Unterschiede gab es bei den körperlichen Beeinträchtigungen. Nahm man die Intervention mit kognitiver Verhaltenstherapie zum Maßstab, schnitt die HDP nicht besser, aber auch nicht schlechter ab. Beide Interventionen erwiesen sich als wirksam, wobei es aber Vorteile für die HDP bei Studienteilnehmern mit höherer Bildung gab.

„Eine internetbasierte Intervention zur Selbsthilfe bei der Bewältigung chronischer Schmerzen, basierend auf Positiver Psychologie, hat sich als klinisch nützlich erweisen“, folgern Peters und Kollegen aus ihren Ergebnissen. Therapeuten seien nicht erforderlich, was eine weite Verbreitung erleichtern sollte. Ob die HDP als einzelne oder als Zusatzbehandlung am wirksamsten einzusetzen sei, müsse noch näher untersucht werden.

(rb)

Quelle:

Basierend auf: „,Gynäkologische Infektionen“ von Prof. Dr. Klaus Friese, Bad Trissl, GynUpdate in Berlin, 25./26. November 2016

\section{Anhaltender Prophylaxeerfolg: Elektroakupunktur punktet bei Migräne}

\section{Mithilfe einer Elektroakupunktur gelingt es bei Patienten mit Migräne ohne Aura, die Häufigkeit der Attacken langanhaltend zu senken. Außerdem wird die Migränedauer verkürzt, wie aus einer chinesischen randomisierten Studie hervorgeht.}

1) Nach Angaben von Dr. Ling Zhao von der Universität für Traditionelle Chinesische Medizin (TCM) in Chengdu/China und ihren Kollegen war bisher unklar, ob in der Migräneprophylaxe eine Akupunktur an den klassischen Punkten einer Kontrollmethode, bei der
Nichtakupunkturpunkte verwendet werden, überlegen ist. Um das zu prüfen, führten die TCM-Experten eine dreiarmige kontrollierte Studie durch, an der als weitere Kontrollgruppe Patienten ohne jegliche Intervention teilnahmen, denen jedoch eine kostenlose
Akupunktur für die Zeit nach der Studie zugesagt worden war.

An der Studie nahmen insgesamt 242 Patienten ( 18 bis 65 Jahre) mit Migräne ohne Aura teil. Die Zahl der Migräneattacken lag zwischen zwei und acht pro Monat. Die Akupunktur mithilfe von Nadeln in zwei Gruppen erfolgte innerhalb von vier Wochen an jeweils fünf Tagen pro Woche. Primärer Endpunkt der Studie war die Veränderung der Häufigkeit der Migräneattacken bis zur Woche 16. Als sekundäre Endpunk- 